\title{
Prevalence of, and risk factors for, diabetes and prediabetes in Bangladesh: Evidence from the national survey using a multilevel Poisson regression model with a robust variance
}

Mohammad Bellal Hossain*a, Md. Nuruzzaman Khan ${ }^{b}$, John C. Oldroyd ${ }^{c}$, Juwel Ranade, Dianna J. Magliago ${ }^{f}$, Enayet K. Chowdhury ${ }^{\mathrm{fg}}$, Md Nazmul Karim ${ }^{\mathrm{e}}$, Rakibul M. Islam ${ }^{\mathrm{ef}}$

${ }^{a}$ Department of Population Sciences, University of Dhaka, Dhaka, Bangladesh.

${ }^{\mathrm{b}}$ Department of Population Sciences, Jatiya Kabi Kazi Nazrul Islam University, Mymensingh, Bangladesh.

'School of Behavioral and Health Sciences, Australian Catholic University, Fitzroy, Victoria, Australia

${ }^{\mathrm{d}}$ Department of Public Health, School of Health and Life Sciences, North South University, Dhaka, Bangladesh

${ }^{\text {e }}$ South Asia Institute for Social Transformation (SAIST), Dhaka, Bangladesh

${ }^{f}$ Department of Epidemiology and Preventive Medicine, School of Public Health and Preventive Medicine, Monash University, Melbourne, Australia.

${ }^{\mathrm{g}}$ School of Public Health, Curtin University, Perth, Western Australia, Australia

* Corresponding author:

Mohammad Bellal Hossain, PhD

Professor

Department of Population Sciences, University of Dhaka

Dhaka-1000, Bangladesh

Email: bellal@du.ac.bd; rakib.islam@monash.edu 


\section{Abstract}

Background: To estimate the age-standardized prevalence of diabetes and prediabetes and identify factors associated with these conditions at individual, household, and community levels.

Methods: Data from 11952 Bangladeshi adults aged 18-95 years available from the most recent Bangladesh Demographic and Health Survey 2017-18 were used. Anthropometric measurements and fasting blood glucose samples were taken as part of the survey. Prevalence estimates of diabetes and prediabetes were age-standardized with direct standardization, and risk factors were identified using multilevel mix-effects Poisson regression models with robust variance.

Results: The overall age-standardised prevalence of diabetes was $9.2 \%(95 \% \mathrm{Cl} 8.7-9.7)$ (men: 8.8\%, women: 9.6\%), and prediabetes was $13.3 \%(95 \% \mathrm{Cl} 12.7-13.9)$ (men: $13.0 \%$, women: $13.6 \%$ ). Among people with diabetes, $61.5 \%$ were unaware that they had the condition. $35.2 \%$ were taking treatment regularly, and only $30.4 \%$ of them had controlled diabetes. Factors associated with an increased prevalence of having diabetes were increasing age, male, overweight/obesity, hypertension, being in the highest wealth quintile, and living in the Dhaka division. People currently employed and living in the Rangpur division were less likely to have diabetes than those currently not employed and living in the Barishal division.

Conclusions: Diabetes and prediabetes affect a substantial proportion (over one-quarter) of the Bangladeshi adult population. Continuing surveillance and effective prevention and control measures, focusing on obesity reduction and hypertension management, are urgently needed.

Keywords: Diabetes Mellitus Type 2, Prediabetic State, Risk Factors, Bangladesh

\section{Introduction}

Diabetes mellitus remains a significant contributor to the global burden of disease (1). People with diabetes have an increased risk of developing several serious life-threatening micro-and macro-vascular complications resulting in higher medical care costs, reduced quality of life, and increased mortality (2). The International Diabetes Federation (IDF) has estimated that 463 million adults live with diabetes worldwide in 2019, with a projected increase to 700 million by 2045 (3). Seventy-nine percent of those with diabetes live in lowand middle-income countries (LMICS) (4). It is projected that diabetes cases will increase by $74 \%$ in Southeast Asian countries in the next two decades, from 88 million in 2019 to 153 million by 2045 (4).

In Bangladesh, 8.4 million adults lived with diabetes in 2019 and projected to be almost double (15.0 million) by 2045 (4). Studies, including a systematic review and meta-analysis, and national survey reports, showed that the prevalence of diabetes among adults had increased substantially in Bangladesh, from 5\% in 2001 to $14 \%$ in 2017(5-8). Several 
studies were also conducted using BDHS 2011 data, showing that people with older age, overweight/obesity, hypertension, and higher socioeconomic status (e.g., education level and wealth status) were associated with increasing likelihoods of diabetes (9-12). However, these studies are relatively old and overlooked the prevalence of, and risk factors for, prediabetes. In 2019, it was estimated that 3.8 million people had prediabetes in Bangladesh, which is a major challenge for the health system in Bangladesh when added with existing cases of diabetes (4).

Thus, it is necessary to study the age-standardized prevalence of and risk factors for diabetes and prediabetes in Bangladeshi adults using the latest Bangladesh Demographic and Health Survey (BDHS) 2017-18. To our knowledge, Kibria (13) has published an article using BDHS 2017-18 with significant flaws, including inconsistent sample size compared to the BDHS 2017-2018 published report and inappropriate age-standardization. Besides, Kibria (13) did not estimate prediabetes and estimated the odds ratio for diabetes using logistic regression, which is not robust when the outcome is common $(14,15)$. Based on these limitations, we aimed to estimate the age-standardized prevalence of diabetes and prediabetes in Bangladeshi adults aged 18 years and older using the latest BDHS. We also investigated factors associated with diabetes and prediabetes in Bangladeshi adults using a multilevel Poisson regression model with robust variance. Results are examined in detail according to the individual, household, and community-level characteristics.

\section{Materials and methods \\ Study population and data collection}

Bangladesh, a Southeast Asian country, currently has a 111 million population aged 18 years and older (16). In 2017-18, the National Institute of Population Research and Training (NIPORT), the Ministry of Health and Family Welfare, Bangladesh, conducted the second BDHS survey of its kind that collected data on blood pressure, fasting blood glucose (FBG) biomarker measurements, and relevant information in addition to socio-demographic characteristics (7).

The BDHS is a nationally representative survey conducted using a two-stage stratified sample of households, including strata for rural and urban areas. Detail survey sampling and the data collection procedure have been published in the BDHS survey report (7). The primary sampling units (PSUs), each containing 120 households on average, were taken from the most recent 2011 Bangladesh census enumeration areas. In BDHS 2017-18, a total of 675 PSUs was selected with probability proportional to PSU size; however, 672 PSUs were included (three PSUs were not sampled due to flooding), of which 192 and 480 were from urban and rural areas, respectively. In the second stage, 30 households per PSU were selected using systematic random sampling to provide statistically reliable estimates of health outcomes for the country as a whole for each of the eight divisions and urban and rural areas separately. Of the 20,160 selected households, interviews were completed in 19,457 households with an overall $96.5 \%$ household response rate (7). Of these, one-fourth of the households (4864) were selected for the collection of biomarkers. A total of 14,704 (8013 women, 6691 men) respondents aged 18+ were available in the 4864 selected households for blood glucose measurement. However, 12,100 (6919 women, 5181 men) 
medRxiv preprint doi: https://doi.org/10.1101/2021.01.26.21250519; this version posted August 4, 2021. The copyright holder for this preprint

(which was not certified by peer review) is the author/funder, who has granted medRxiv a license to display the preprint in perpetuity.

All rights reserved. No reuse allowed without permission.

respondents aged 18 years and older had their blood glucose tested (82.3\% response rate) (Figure 1).

Figure 1: Schematic representation of the sampling procedure of the Bangladesh Demographic and Health Survey, 2017-18

\section{Definition of diabetes and prediabetes}

The primary outcomes in this study were diabetes and prediabetes calculated based on the fasting plasma glucose (FPG) level (7). The HemoCue Glucose 201 DM system with plasma conversion was used to test a drop of capillary blood obtained from consenting eligible respondents from the middle or ring finger. The system automatically converted the fasting whole blood glucose measurements taken in the survey to FPG equivalent values. The respondents were asked not to eat or drink anything other than plain water for at least 8 hours before testing. The details on blood sample collection have been described in the BDHS survey report (7). The World Health Organization (WHO) criteria for diabetes and prediabetes classification were used (17). Diabetes was defined as an FPG level greater than or equal to $7.0 \mathrm{mmol} / \mathrm{L}$ or self-reported diabetes medication use. Prediabetes was defined as an FPG level from $6.1 \mathrm{mmol} / \mathrm{L}$ to $6.9 \mathrm{mmol} / \mathrm{L}$, without medication (17).

\section{Explanatory variables}

Explanatory variables included individual, household, and community-level factors. Individual-level factors included were participants' age, sex, BMI, educational level, working status, and hypertension. The BMI was categorized based on Asian cut-off as suggested by the WHO expert consultation due to the high risk of type 2 diabetes and cardiovascular disease in Asian people at lower BMIs than the existing WHO cut-off (18). The presence of hypertension was defined as a systolic blood pressure $\geq 140 \mathrm{mmHg}$ and/or a diastolic blood pressure $\geq 90 \mathrm{mmHg}$, and/or currently on treatment with antihypertensive medication (19). The household wealth quintile (lowest to highest) was the household-level factor. It was derived from the household wealth index reported in the BDHS, which was constructed using principal component analysis from household's durable and non-durable assets (e.g., televisions, bicycles, sources of drinking water, sanitation facilities, and construction materials) (20). Community-level factors included were the place of residence and administrative divisions of the country.

\section{Statistical analysis}

The crude prevalence of diabetes and prediabetes were estimated, allowing for the complex survey design and survey sampling weights. To account for different age distributions between groups and over time, we age-standardized estimates to the 2011 Census population of Bangladesh using the direct method, with age categories of 18-34, 35-39, 40$44,45-49,50-54,55-59,60-64$ and $\geq 65$ years. Differences between continuous and categorical variables were tested using the Mann-Whitney test and chi-square tests, respectively. We used a multilevel Poisson regression model with a robust variance to identify factors associated with diabetes and prediabetes, and results were presented as a prevalence ratio (PR) with a 95\% confidence interval $(\mathrm{Cl})$. We used this model since the odds ratio estimated using logistic regression from a cross-sectional study may significantly overestimate relative risk when the outcome is common $(14,15)$. Secondly, in the case of 
convergence failure with the log-binomial model, Poisson regression with a robust variance performs better in estimating the prevalence ratio from a cross-sectional study (21).

Furthermore, in the BDHS, individuals were nested within the household; households were nested within the PSU/cluster. Hence, our multilevel mixed-effects Poisson regression model accounts for these multiple hierarchies and dependency in data and the problem of overestimation (22). With progressive model-building techniques, four models were run separately for diabetes and prediabetes for different confounding factors at the individual, household, and community levels. Model 1 was run without confounding factors to determine the cluster level variation of diabetes and prediabetes in Bangladesh. Model 2 and 3 were adjusted for individual, and individual plus household level factors, respectively. Model 4 was the final model that included individual, household, and community-level factors simultaneously. The Intra-Class Correlation (ICC), Akaike Information Criteria (AIC), and Bayesian Information Criteria (BIC) were used to assess model performance. All statistical tests were two-sided, and a p-value $<0.05$ was considered statistically significant. The study was designed and reported following strengthening the Reporting of Observational Studies in Epidemiology (STROBE) guidelines (23). All analyses were performed using statistical software packages Stata (version 15·10; Stata Corp LP, College Station, Texas).

\section{Ethical approval}

This study is a secondary analysis of publicly available household survey data. Thus, we did not require any ethical approval for this study. However, institutional review boards (IRBs) at ICF and the Bangladesh Medical Research Council (BMRC) approved the survey methodology, biomarker measurements, and other survey instruments. In addition, the BDHS 2017-18 datasets are publicly available (https://dhsprogram.com/methodology/survey/survey-display-536.cfm), and we received authorization from the DHS to use the datasets.

\section{Results}

Of 12,100 participants who provided FBG, 11,952 were included in the analysis (Figure 1). The median (IQR) age of the participants was 36 (24) years (Table 1). Of the study participants, $57.1 \%(6,826)$ were female, $26.6 \%(3,179)$ lived in urban areas, $40.1 \%(4,794)$ were overweight/obese and $27.4 \%(3,274)$ had hypertension. People with diabetes were significantly older than those without $(p<0.001)$, they were significantly more likely to be overweight or obese $(p<0.001)$, more likely to be hypertensive $(p<0.001)$. Furthermore, people with diabetes were more likely to live in urban areas and come from a household with higher wealth quintiles ( $p<0.001$ ) (Table 1 ).

Table 1: Characteristics of the study population aged $\geq 18$ years in Bangladesh, BDHS 2017-18

\begin{tabular}{|c|c|c|c|c|}
\hline \multirow[b]{2}{*}{ Characteristics } & \multicolumn{3}{|c|}{$N(\%)^{a}$} & \multirow[b]{2}{*}{ P-value ${ }^{b}$} \\
\hline & $\begin{array}{r}\text { All } \\
(\mathrm{N}=11,952)\end{array}$ & $\begin{array}{r}\text { No. without diabetes } \\
\qquad(\mathrm{N}=10,778)\end{array}$ & $\begin{array}{r}\text { No. with diabetes } \\
(N=1,174)\end{array}$ & \\
\hline \multicolumn{5}{|l|}{ Individual level } \\
\hline Age in years, Median (IQR) & $39(24)$ & $35(23)$ & $46(23)$ & $<0.001$ \\
\hline $18-34$ & $5,391(45.1)$ & $5,114(47.4)$ & $277(23.6)$ & \\
\hline $35-39$ & $1,371(11.5)$ & $1,233(11.4)$ & $138(11.8)$ & \\
\hline $40-44$ & $1,047(8.7)$ & $916(8.5)$ & $131(11.2)$ & \\
\hline $45-49$ & $994(8.3)$ & $861(8.0)$ & $133(11.3)$ & \\
\hline $50-54$ & $672(5.6)$ & $556(5.2)$ & $115(9.8)$ & \\
\hline
\end{tabular}




\begin{tabular}{|c|c|c|c|c|}
\hline $55-59$ & $676(5.7)$ & $577(5.4)$ & $100(8.5)$ & \\
\hline $60-64$ & $675(5.7)$ & $563(5.2)$ & $112(9.5)$ & \\
\hline$\geq 65$ & $1,126(9.42)$ & $958(8.9)$ & $168(14.3)$ & \\
\hline \multicolumn{5}{|l|}{ Sex } \\
\hline Men & $5,126(42.9)$ & $4,589(42.6)$ & $537(45.8)$ & \multirow{2}{*}{0.550} \\
\hline Women & $6,826(57.1)$ & $6,189(57.4)$ & $637(54.2)$ & \\
\hline \multicolumn{5}{|l|}{ Body Mass Index $\left(\mathrm{kg} / \mathrm{m}^{2}\right)$} \\
\hline Underweight $(<18.5)$ & $2,067(17.3)$ & $1,938(18.0)$ & $129(11.0)$ & \multirow{4}{*}{$<0.001$} \\
\hline Normal weight (18.5-23.0) & $5091(42.6)$ & $4696(43.6)$ & $395(33.7)$ & \\
\hline Overweight (23.0-27.5) & $3532(29.6)$ & $3107(28.8)$ & $425(36.2)$ & \\
\hline Obese $(\geq 27.5)$ & $1262(10.5)$ & $1037(9.6)$ & $225(19.1)$ & \\
\hline \multicolumn{5}{|l|}{ Level of education } \\
\hline No education/preschool & $3,033(25.4)$ & $2,736(25.4)$ & $296(25.2)$ & \multirow{4}{*}{0.937} \\
\hline Primary education & $3,590(30.0)$ & $3,225(29.9)$ & $365(31.1)$ & \\
\hline Secondary education & $3,539(29.6)$ & $3,203(29.7)$ & $337(28.7)$ & \\
\hline Higher education & $1,790(15.0)$ & $1,614(15.0)$ & $176(15.0)$ & \\
\hline \multicolumn{5}{|l|}{ Currently working } \\
\hline Yes & $4,621(38.7)$ & $6,695(62.1)$ & $636(54.1)$ & \multirow{2}{*}{$<0.001$} \\
\hline No & $7,331(61.3)$ & $4,083(37.9)$ & $538(45.9)$ & \\
\hline \multicolumn{5}{|l|}{ Hypertension } \\
\hline Yes & $3,416(28.6)$ & $2,865(26.6)$ & $551(46.9)$ & \multirow{2}{*}{$<0.001$} \\
\hline No & $8,536(71.4)$ & $7,913(73.4)$ & $623(53.1)$ & \\
\hline \multicolumn{5}{|l|}{ Household level } \\
\hline \multicolumn{5}{|l|}{ Wealth quintile } \\
\hline Lowest & $2,311(19.3)$ & $2,183(20.3)$ & $128(10.9)$ & \multirow{5}{*}{$<0.001$} \\
\hline Second & $2,354(19.7)$ & $2,213(20.5)$ & $141(12.0)$ & \\
\hline Middle & $2,466(20.6)$ & $2,272(21.1)$ & $194(16.5)$ & \\
\hline Fourth & $2,377(19.9)$ & $2,111(19.6)$ & $266(22.7)$ & \\
\hline Highest & $2,444(20.5)$ & $1,999(18.5)$ & 445 (37.9) & \\
\hline \multicolumn{5}{|l|}{$\begin{array}{l}\text { Community level } \\
\text { Place of residence }\end{array}$} \\
\hline Urban & $3,179(26.6)$ & $2,764(25.6)$ & $416(35.4)$ & \multirow[b]{2}{*}{$<0.001$} \\
\hline Rural & $8,773(73.4)$ & $8,014(74.4)$ & $758(64.6)$ & \\
\hline \multicolumn{5}{|l|}{ Administrative division } \\
\hline Barishal & $660(5.5)$ & $597(5.5)$ & $62(5.3)$ & \multirow{8}{*}{$<0.001$} \\
\hline Chattogram & $2,053(17.2)$ & $1,828(17.0)$ & $224(19.1)$ & \\
\hline Dhaka & $2,767(23.2)$ & $2,373(22.0)$ & $394(33.6)$ & \\
\hline Khulna & $1,489(12.5)$ & $1,368(12.7)$ & $121(10.3)$ & \\
\hline Mymensingh & $973(8.1)$ & $897(8.3)$ & $77(6.5)$ & \\
\hline Rajshahi & $1,727(14.4)$ & $1,590(14.8)$ & $138(11.7)$ & \\
\hline Rangpur & $1,503(12.6)$ & $1,419(13.2)$ & $84(7.2)$ & \\
\hline Sylhet & $780(6.5)$ & $706(6.5)$ & $74(6.3)$ & \\
\hline
\end{tabular}

The crude and age-standardized prevalence of diabetes and prediabetes by individual, household, and community-level characteristics are presented in Table 2. The overall agestandardized prevalence of diabetes was $9.2 \%(95 \% \mathrm{Cl}, 8.7-9.7 \%)$ with comparable estimates for men: $8.8 \%, 95 \% \mathrm{Cl} 8.1-9.6$, and women: $9.6 \%, 95 \% \mathrm{Cl}$ 8.9-10.3. The age-standardized diabetes prevalence was higher in urban $(11.8 \%, 95 \% \mathrm{Cl} 10.9-12.7)$ than in rural residents (7.9\%, 95\% Cl 7.3-8.5). Prevalence of diabetes was highest in people who were obese $(18.4 \%$, $95 \% \mathrm{Cl} 16.3-20.5)$, hypertensive $(13.7 \%, 95 \% \mathrm{Cl} 12.3-15.0)$, in the highest wealth quintile $(16.5 \%, 95 \% \mathrm{Cl} 15.9-17.9)$, and living in the Dhaka division $(15.0 \%, 95 \% \mathrm{Cl} 13.3-16.7)$ compared to respective reference categories.

Table 2: Crude and age-standardized prevalence of diabetes and prediabetes in adults aged $\geq 18$ years in Bangladeshi population, BDHS 2017-18 
medRxiv preprint doi: https://doi.org/10.1101/2021.01.26.21250519; this version posted August 4, 2021. The copyright holder for this preprint (which was not certified by peer review) is the author/funder, who has granted medRxiv a license to display the preprint in perpetuity.

All rights reserved. No reuse allowed without permission.

\begin{tabular}{|c|c|c|c|c|}
\hline & Crude prevalence & $\begin{array}{r}\text { Standardized } \\
\text { prevalence }\end{array}$ & Crude prevalence & $\begin{array}{r}\text { Standardized } \\
\text { prevalence }\end{array}$ \\
\hline Overall & $9.8(9.1-10.6)$ & $9.2(8.7-9.7)$ & $14.2(13.3-15.1)$ & $13.3(12.7-13.9)$ \\
\hline \multicolumn{5}{|l|}{ Individual level } \\
\hline \multicolumn{5}{|l|}{ Age (years) } \\
\hline $18-34$ & $5.1(4.4-5.9)$ & $5.1(4.53-5.70)$ & $12.7(11.5-14.0)$ & $11.5(10.6-12.4)$ \\
\hline $35-39$ & $10.0(8.4-12.0)$ & $10.0(8.4-11.5)$ & $15.6(13.5-17.9)$ & $15.2(13.3-17.1)$ \\
\hline $40-44$ & $12.5(10.4-15.1)$ & $11.8(9.8-13.7)$ & $15.6(13.1-18.5)$ & $14.9(12.7-17.1)$ \\
\hline $45-49$ & $13.4(11.2-15.9)$ & $12.9(10.8-14.9)$ & $15.7(13.4-18.3)$ & $15.4(13.2-17.6)$ \\
\hline $50-54$ & $17.2(14.2-20.7)$ & $16.4(13.6-19.2)$ & $14.3(11.6-17.6)$ & $14.7(12.1-17.4)$ \\
\hline $55-59$ & $14.8(12.0-18.0)$ & $15.9(13.2-18.6)$ & $15.7(12.9-18.9)$ & $15.3(12.6-18.0)$ \\
\hline $60-64$ & $16.5(13.7-19.8)$ & $15.3(12.6-18.0)$ & $15.7(12.8-19.2)$ & $15.4(12.7-18.1)$ \\
\hline$\geq 65$ & $14.9(12.6-17.5)$ & $14.8(12.8-16.9)$ & $14.9(12.5-17.7)$ & $14.7(12.7-16.8)$ \\
\hline \multicolumn{5}{|l|}{ Sex } \\
\hline Men & $10.5(9.5-11.5)$ & $8.8(8.1-9.6)$ & $14.0(12.8-15.2)$ & $13.0(12.1-13.9)$ \\
\hline Women & $9.3(8.5-10.2)$ & $9.6(8.9-10.3)$ & $14.4(13.3-15.5)$ & $13.6(12.8-14.5)$ \\
\hline \multicolumn{5}{|l|}{$\begin{array}{l}\text { Body Mass Index } \\
\left(\mathrm{kg} / \mathrm{m}^{2}\right)\end{array}$} \\
\hline Underweight $(<18.5)$ & $6.2(5.0-7.6)$ & $5.3(4.3-6.3)$ & $12.5(10.8-14.4)$ & $11.9(10.4-13.3)$ \\
\hline $\begin{array}{l}\text { Normal weight (18.5- } \\
23.0)\end{array}$ & $7.8(6.9-8.7)$ & $7.3(6.6-8.0)$ & $12.9(11.7-14.1)$ & $12.2(11.3-13.1)$ \\
\hline Overweight (23.0-27.5) & $12.0(10.8-13.4)$ & $11.6(10.5-12.6)$ & $15.3(13.8-16.8)$ & $14.1(12.9-15.2)$ \\
\hline Obese $(\geq 27.5)$ & $17.8(15.6-20.3)$ & $18.4(16.3-20.5)$ & $19.4(16.9-22.0)$ & $18.5(16.3-20.7)$ \\
\hline \multicolumn{5}{|l|}{ Level of education } \\
\hline $\begin{array}{l}\text { No education, } \\
\text { preschool }\end{array}$ & $9.7(8.6-11.1)$ & $6.9(8.4-10.5)$ & $14.8(13.2-16.6)$ & $13.0(11.3-14.8)$ \\
\hline Primary & $10.2(9.0-11.5)$ & $9.3(8.8-10.7)$ & $13.4(12.1-14.8)$ & $12.7(11.6-13.8)$ \\
\hline Secondary & $9.5(8.4-10.7)$ & $11.7(8.6-10.5)$ & $14.3(12.8-15.8)$ & $13.6(12.4-14.8)$ \\
\hline Higher education & $9.8(8.3-11.6)$ & $13.1(8.6-11.2)$ & $14.4(12.5-16.5)$ & 15.7 (13.9-17.5) \\
\hline \multicolumn{5}{|l|}{ Currently working } \\
\hline Yes & $8.7(7.9-9.5)$ & $8.1(7.5-8.7)$ & $13.5(12.4-14.5)$ & $12.5(11.8-13.3)$ \\
\hline No & $11.6(10.5-12.9)$ & $11.8(10.9-12.8)$ & $15.3(14.0-16.8)$ & $14.6(13.6-15.7)$ \\
\hline \multicolumn{5}{|l|}{ Hypertension } \\
\hline Yes & $16.4(15.0-18.0)$ & $13.7(12.3-15.0)$ & $14.9(13.5-16.5)$ & $13.4(12.0-14.8)$ \\
\hline No & $7.3(6.6-8.1)$ & $7.4(6.8-8.0)$ & $13.9(12.9-15.0)$ & $13.2(12.5-13.9)$ \\
\hline \multicolumn{5}{|l|}{$\begin{array}{l}\text { Household level } \\
\text { Wealth quintile }\end{array}$} \\
\hline Lowest & $5.5(4.4-6.9)$ & $5.3(4.4-6.2)$ & $11.8(10.1-13.8)$ & $11.5(10.2-12.8)$ \\
\hline Second & $6.0(5.0-7.2)$ & $5.6(4.7-6.6)$ & $10.3(8.8-11.9)$ & $10.0(8.8-11.2)$ \\
\hline Middle & $7.8(6.7-9.2)$ & $7.6(6.5-8.6)$ & $11.7(10.2-13.6)$ & $11.2(9.8-12.3)$ \\
\hline Fourth & $11.2(9.7-12.9)$ & $10.4(9.1-11.6)$ & $16.2(14.4-18.2)$ & $14.6(13.2-16.1)$ \\
\hline Highest & $18.2(16.5-20.1)$ & $16.5(15.9-17.9)$ & $20.6(18.7-22.7)$ & $18.6(17.1-20.1)$ \\
\hline \multirow{2}{*}{\multicolumn{5}{|c|}{$\begin{array}{l}\text { Community level } \\
\text { Place of residence }\end{array}$}} \\
\hline & & & & \\
\hline Urban & $13.1(11.8-14.5)$ & $11.8(10.9-12.7)$ & $17.7(16.0-19.6)$ & $15.1(14.0-16.2)$ \\
\hline Rural & $8.6(7.8-9.6)$ & $7.9(7.3-8.5)$ & $12.9(11.8-14.0)$ & $12.3(11.6-13.0)$ \\
\hline \multicolumn{5}{|l|}{ Administrative division } \\
\hline Barishal & $9.4(7.5-11.8)$ & $9.2(7.7-10.8)$ & $16.3(13.6-19.5)$ & $16.6(14.5-18.7)$ \\
\hline Chattogram & $10.9(9.0-13.2)$ & $10.9(9.4-12.3)$ & $14.6(12.7-16.8)$ & $14.4(12.7-16.1)$ \\
\hline Dhaka & $14.2(12.3-16.5)$ & $15.0(13.3-16.7)$ & $22.0(19.3-25.1)$ & $22.8(20.7-24.8)$ \\
\hline Khulna & $8.2(6.8-9.7)$ & $8.0(6.8-9.2)$ & $10.6(8.8-12.9)$ & $10.0(8.6-11.5)$ \\
\hline Mymensingh & $7.9(6.3-9.9)$ & $7.8(6.3-9.2)$ & $12.2(10.5-14.2)$ & $12.3(10.5-14.0)$ \\
\hline Rajshahi & $8.0(6.4-9.8)$ & $8.2(6.9-9.5)$ & 9.7 (7.8-11.9) & $9.3(7.9-10.7)$ \\
\hline Rangpur & $5.6(4.4-7.0)$ & $5.6(4.5-6.7)$ & $9.0(7.3-11.1)$ & $9.1(7.7-10.6)$ \\
\hline Sylhet & $9.4(7.3-12.2)$ & $9.6(8.2-11.1)$ & $12.6(10.5-15.0)$ & $12.6(10.8-14.3)$ \\
\hline
\end{tabular}

BDHS= Bangladesh Demographic and Health Survey; $\mathrm{Cl}=$ confidence interval.

Data are $\%(95 \% \mathrm{Cl})$. All Cls were logit transformed. Standardized to the 2011 Bangladesh Census population by age for the total population.

Diabetes is defined as a fasting blood glucose level $\geq 7.0 \mathrm{mmol} / \mathrm{L}$ or self-reported diabetes medication use. Prediabetes is defined as a fasting blood glucose level from $6.1 \mathrm{mmol} / \mathrm{L}$ to $6.9 \mathrm{mmol} / \mathrm{L}$, without medication.

The overall age-standardized prevalence of prediabetes was $13.3 \%$ (95\% Cl, 12.7-13.9), with a similar prevalence in women $(13.6 \%, 95 \% \mathrm{Cl} 12.8-14.5)$ and in men $(13.0 \%, 95 \% \mathrm{Cl} 12.1-$ 
13.9). The age-standardized prevalence estimates of prediabetes were higher in people who were obese $(18.5 \%, 95 \% \mathrm{Cl} 16.3-20.7)$, in the highest wealth quintile $(18.6 \%, 95 \% \mathrm{Cl} 17.1$ $20.1)$, and were living in the Dhaka division $(22.8 \%, 95 \% \mathrm{Cl} 20.7-24.8)$ (Table 2). Three out of five $(61.5 \%, 95 \% \mathrm{Cl} 57.9-64.9)$ people living with diabetes were unaware of their condition. One-third $(35.2 \%, 95 \% \mathrm{Cl} 32.0-38.5)$ received appropriate treatment, and only $30.4 \%(95 \% \mathrm{Cl}$ 26.0-35.2) of them had controlled diabetes.

Each of the four mixed-effects multilevel Poisson models was run to identify factors associated with diabetes and prediabetes. We compared intra-class correlation (ICC), Akaike's information criterion (AIC), and Bayesian information criterion (BIC) to select the best fitting model: the preferred model having the smallest ICC, AIC and BIC. According to these indicators, Model 4 (including individual, household, and community-level factors) had the best fitting model. Model 1 (crude) produced an ICC of $30.52 \%$ and $42.54 \%$ for diabetes and prediabetes, respectively (Table 3). This result indicates the degree of the variance seen across clusters without taking other factors into account. However, the ICC was reduced to $12.42 \%$ for diabetes and $14.89 \%$ for prediabetes once individual, household, and community-level factors were included in the final model.

Table 3: Results from the random intercept model (measure of variation) at cluster/community level for diabetes and prediabetes in adults aged $\geq 18$ years in Bangladeshi population, BDHS 2017-18

\begin{tabular}{|c|c|c|c|c|c|c|c|c|}
\hline \multirow{2}{*}{$\begin{array}{l}\text { Random effects (measure of } \\
\text { variation for diabetes) }\end{array}$} & \multicolumn{6}{|c|}{ Diabetes $^{ \pm}$} & \multicolumn{2}{|c|}{ Prediabetes $^{ \pm}$} \\
\hline & Model 1 & Model 2 & Model 3 & Model 4 & Model 1 & Model 2 & Model 3 & Model 4 \\
\hline Cluster-level variance (SE) ${ }^{\mathrm{b}}$ & $\begin{array}{r}0.31 \\
(0.04)\end{array}$ & $\begin{array}{r}0.23 \\
(0.04)\end{array}$ & $\begin{array}{r}0.16 \\
(0.04)\end{array}$ & $\begin{array}{r}0.13 \\
(0.04)\end{array}$ & $\begin{array}{r}0.28 \\
(0.03)\end{array}$ & $\begin{array}{r}0.26 \\
(0.03)\end{array}$ & $\begin{array}{r}0.21 \\
(0.03)\end{array}$ & $\begin{array}{r}0.15 \\
(0.03)\end{array}$ \\
\hline Intra-class correlation (ICC, \%) & $30.52 \%$ & $22.57 \%$ & $15.53 \%$ & $12.47 \%$ & $42.54 \%$ & $25.27 \%$ & $20.54 \%$ & $14.89 \%$ \\
\hline Explained variance (PCV)(\%) & Reference & $25.81 \%$ & $48.38 \%$ & $61.29 \%$ & Reference & $7.14 \%$ & $25.00 \%$ & $46.42 \%$ \\
\hline Model summary & & & & & & & & \\
\hline AIC & 7695.98 & 7264.89 & 7176.57 & 7156.65 & 9857.34 & 9849.54 & 9800.16 & 9733.91 \\
\hline $\mathrm{BIC}$ & 7710.79 & 7398.19 & 7339.49 & 7378.80 & 9872.15 & 99.82 .83 & 9963.07 & 9956.06 \\
\hline $\begin{array}{l}{ }^{ \pm} \text {Models are based on mul } \\
{ }^{a} \text { We assume that the withi } \\
\text { between cluster-level varia } \\
\text { with a similar one in which } \\
\text { Model } 1 \text { is the null model, } \\
\text { Model } 2 \text { is adjusted for ind } \\
\text { Model } 3 \text { is adjusted for ind } \\
\text { Model } 4 \text { is adjusted for ind } \\
\text { The results of all models ar } \\
4 . \\
\text { AIC= Akaike's Information } \\
\text { Criteria; SE = Standard Erro }\end{array}$ & $\begin{array}{l}\text { evel mixed-e } \\
\text { duster-level } \\
\text { ce estimates } \\
\text { ndom effec } \\
\text { daseline mo } \\
\text { dual level fa } \\
\text { dual level pl } \\
\text { dual level pl } \\
\text { presented in } \\
\text { iterion; BDH }\end{array}$ & $\begin{array}{l}\text { re reporte } \\
\text { were cons } \\
\text { without } \\
\text { ors } \\
\text { s househol } \\
\text { s househol } \\
\text { upplemen } \\
\text { = Banglade }\end{array}$ & $\begin{array}{l}\text { regression } \\
\text { s are equa } \\
{ }^{b} \text { Significan } \\
\text { ained to ze } \\
\text { y determin } \\
\text { evel factor } \\
\text { evel plus c } \\
\text { ry Table } 1 \\
\text { Demograp }\end{array}$ & $\begin{array}{l}\text { e of rando } \\
\text { o. } \\
\text { nt variable } \\
\text { nd the rest } \\
\text { ic and Hea }\end{array}$ & $\begin{array}{l}\text { vel factors } \\
\text { ts of the fin } \\
\text { Survey; B }\end{array}$ & $\begin{array}{l}\text { nodel ar } \\
=\text { Bayesia }\end{array}$ & $\begin{array}{l}\text { esented ir } \\
\text { formation }\end{array}$ & $\begin{array}{l}\text { only } \\
\text { odel }\end{array}$ \\
\hline
\end{tabular}

The results for model 4 are shown in Table 4, while the results for all other models are shown in Supplementary Table 1. The final model showed that diabetes was associated with age, sex, BMI, employment status, hypertension, wealth quintile, and administrative division of the country but not with the place of residence (urban/rural) or level of education. Compared with individuals aged 18 to 34 years, individuals aged 40 to 49 years were over two times more likely to have diabetes, while individuals aged $\geq 50$ years were about three times more likely to have diabetes (Table 4). Men were more likely to have diabetes (PR $1.17,95 \% \mathrm{Cl} 1.01-1.36)$ than women. Diabetes was significantly positively associated with being overweight (PR 1.23, 95\% Cl 1.06-1.43) or obese (PR 1.45, 95\% Cl 1.21-1.75) compared with normal weight, being hypertensive (PR 1.47, 95\% $\mathrm{Cl} 1.30-1.68)$ compared with 
normotensive, belonging to either of the fourth (PR 1.60, 95\% $\mathrm{Cl} 1.23-2.09$ ); or the highest wealth quintile ( $P R 2.21,95 \% \mathrm{Cl} 1.70-2.86)$, compared with the lowest quintile, and living in the Dhaka division (PR 1.32, 95\% $\mathrm{Cl}$ 1.02-1.71) compared with the Barishal division. Individuals currently employed (PR 0.81, 95\% $\mathrm{Cl} 0.69-0.94$ ) and living in the Rangpur division (PR 0.67, 95\% $\mathrm{Cl} 0.50-0.91$ ) were less likely to have diabetes than being employed and living in the Barishal division.

Table 4: Factors associated with diabetes and prediabetes in adults aged $\geq 18$ years in Bangladeshi population, BDHS 2017-18

\begin{tabular}{|c|c|c|}
\hline Characteristics & Diabetes, PR $(95 \% \mathrm{Cl})^{ \pm}$ & Pre-diabetes, PR $(95 \% \mathrm{Cl})^{ \pm}$ \\
\hline \multicolumn{3}{|l|}{ Individual level } \\
\hline \multicolumn{3}{|l|}{ Age in years, (ref: 18-34) } \\
\hline $35-39$ & $1.80(1.45-2.25)$ & $1.19(1.01-1.41)$ \\
\hline $40-44$ & $2.37(1.87-3.00)$ & $1.22(1.01-1.48)$ \\
\hline $45-49$ & $2.39(1.89-3.01)$ & $1.22(1.02-1.45)$ \\
\hline $50-54$ & $3.16(2.45-4.06)$ & $1.12(0.90-1.41)$ \\
\hline $55-59$ & $2.70(2.13-3.43)$ & $1.26(1.01-1.58)$ \\
\hline $60-64$ & $3.11(2.46-3.92)$ & $1.26(1.00-1.59)$ \\
\hline$\geq 65$ & $2.77(2.16-3.55)$ & $1.21(0.99-1.49)$ \\
\hline Sex, (ref: women) & $1.17(1.01-1.36)$ & $0.98(0.87-1.10)$ \\
\hline \multicolumn{3}{|l|}{$\begin{array}{l}\text { Body Mass Index }\left(\mathrm{kg} / \mathrm{m}^{2}\right) \text {, (ref: normal } \\
\text { weight) }\end{array}$} \\
\hline Underweight $(<18.5)$ & $0.83(0.67-1.03)$ & $0.97(0.83-1.13)$ \\
\hline Overweight (23.0-27.5) & $1.23(1.06-1.43)$ & $1.07(0.95-1.21)$ \\
\hline Obese $(\geq 27.5)$ & $1.45(1.21-1.75)$ & $1.23(1.05-1.44)$ \\
\hline \multicolumn{3}{|l|}{ Level of education, (ref: higher education) } \\
\hline No education, preschool & $0.93(0.74-1.18)$ & $1.16(0.95-1.42)$ \\
\hline Primary & $1.16(0.95-1.42)$ & $1.04(0.88-1.24)$ \\
\hline Secondary & $1.08(0.90-1.30)$ & $1.05(0.89-1.23)$ \\
\hline Currently working, (ref: no) & $0.81(0.69-0.94)$ & $1.01(0.90-1.14)$ \\
\hline Hypertension, (ref: no) & $1.47(1.30-1.68)$ & $0.98(0.87-1.10)$ \\
\hline \multicolumn{3}{|l|}{ Household level } \\
\hline \multicolumn{3}{|l|}{ Wealth quintile, (ref: lowest) } \\
\hline Second & $1.04(0.79-1.35)$ & $0.82(0.66-1.03)$ \\
\hline Middle & $1.24(0.96-1.60)$ & $0.94(0.77-1.15)$ \\
\hline Fourth & $1.60(1.23-2.09)$ & $1.16(0.95-1.42)$ \\
\hline Highest & $2.21(1.70-2.86)$ & $1.36(1.10-1.68)$ \\
\hline \multicolumn{3}{|l|}{ Community level } \\
\hline \multicolumn{3}{|l|}{ Administrative division, (ref: Barishal) } \\
\hline Chattogram & $0.98(0.74-1.29)$ & $0.82(0.66-1.03)$ \\
\hline Dhaka & $1.32(1.02-1.71)$ & $1.19(0.94-1.49)$ \\
\hline Khulna & $0.78(0.60-1.01)$ & $0.59(0.46-0.77)$ \\
\hline Mymensingh & $0.94(0.70-1.27)$ & $0.74(0.59-0.93)$ \\
\hline Rajshahi & $0.90(0.68-1.20)$ & $0.57(0.43-0.75)$ \\
\hline Rangpur & $0.67(0.50-0.91)$ & $0.54(0.42-0.71)$ \\
\hline Sylhet & $1.00(0.72-1.38)$ & $0.73(0.56-0.94)$ \\
\hline
\end{tabular}

The fully adjusted model showed that compared with the younger age group, individuals aged between 35 to 49 years were $19 \%$ to $23 \%$ more likely to have prediabetes, and 
medRxiv preprint doi: https://doi.org/10.1101/2021.01.26.21250519; this version posted August 4, 2021. The copyright holder for this preprint

(which was not certified by peer review) is the author/funder, who has granted medRxiv a license to display the preprint in perpetuity.

All rights reserved. No reuse allowed without permission.

individuals aged 55 to 64 years were $26 \%$ more likely to have prediabetes. Being obese (PR $1.23,95 \% \mathrm{Cl} 1.05-1.44$ ) and belonging to the highest wealth quantile (PR 1.36, 95\% $\mathrm{Cl} 1.10$ 1.68) were associated with prediabetes compared with being normal weight and being in the lowest wealth quintile. The respondents living in Khulna, Mymensingh, Rajshahi, Rangpur, and Sylhet divisions were about $25 \%$ to $45 \%$ less likely to have prediabetes than the Barishal division. Sex, level of education, working status, hypertension, and place of residence were not associated with prediabetes.

\section{Discussion}

Diabetes and prediabetes affect a substantial proportion of the Bangladeshi population. Based on data from the latest BDHS 2017-18, over one-quarter of individuals aged 18 years and older had diabetes or prediabetes in Bangladesh, representing more than 19 million individuals in 2020. Factors associated with diabetes were age, sex, BMI, wealth quintile, employment status, hypertension, and administrative division of the country but not the place of residence (urban /rural) or education level. These findings confirm a continuing high burden of diabetes and prediabetes in Bangladesh.

We reported that the prevalence of diabetes is lower than the overall age-adjusted diabetes prevalence of $11.3 \%$ in the Southeast Asian region, estimated by the IDF in 2019(4). The IDF has identified that the countries with the largest numbers of adults with diabetes aged 2079 years in 2019 in the region are China (116 million cases) and India (77 million cases) (4). In 2019, the IDF ranked Bangladesh $10^{\text {th }}$ of countries with the highest number of adults (2079 years) with diabetes (8.4 million cases), and it is expected to be ranked $9^{\text {th }}$ in $2030(4)$, consistent with our estimates. In our analysis, about 1 in 10 adults (18+) had diabetes, representing an estimated over 7.9 million individuals in Bangladesh in 2020. Note that our data included the younger population compared with the IDF estimates; as such, the total number of cases is deflated due to a very low prevalence of diabetes in the younger population. Nevertheless, this large number of diabetes cases in Bangladesh indicates that it is one of the leading countries for diabetes burden in the Southeast Asian region and highlights the urgent need for policies supporting the rollout of diabetes prevention in this country.

Our study undertook an identical methodological approach (e.g., anthropometric measurements and fasting blood samples) to the 2011 study and demonstrated that the prevalence of diabetes had increased markedly in Bangladesh over seven years (5). Similar increasing trends of diabetes have been observed in other Southeast Asian countries (24). However, the extent to which changes in traditional diabetes risk factors can explain the increasing trends in the prevalence of diabetes in this setting needs further investigation. A higher diabetes prevalence suggests that despite greater global awareness of diabetes and interventions for improved non-communicable disease management in primary health care (25), diabetes in Bangladesh is increasing. Furthermore, it suggests that health promotion may be failing in the face of dietary and lifestyle patterns. Thus, more resources are needed to be invested in primary health care to address the prevention of diabetes in Bangladesh.

Our estimates suggest that the prevalence of prediabetes has decreased in Bangladesh in the seven years between 2011 and 2017/18 (5). Prediabetes is important as during this stage, micro-vascular complications occur, often without people knowing they are glucose 
intolerant. The literature shows that up to $40.5 \%$ of individuals with prediabetes convert to diabetes during follow-up (26). A high conversion rate of prediabetes to diabetes is indicative of the potential for an uncontrolled increase in the prevalence of diabetes. The observation that diabetes prevalence has increased but prediabetes has decreased in seven years may indicate higher than expected conversion rates due to rapidly changing environmental conditions (25). There is a well-established relationship between increasing age and risk of diabetes which is consistent with our study $(4,27)$. The implication is that with increasing life expectancy in Bangladesh (current life expectancy at birth 72.3 years) (28), the increasing numbers of older people will result in even more cases and a higher burden of diabetes.

Consistent with our study, the literature shows that obesity is a leading risk factor for type 2 diabetes (29). However, the association between obesity and diabetes is complicated as obesity is also related to socioeconomic status. We observed a significantly higher prevalence of diabetes in the highest wealth quintile than the lowest wealth quintile. A possible explanation is that those in the highest wealth quintile in LMICs use disposable income to purchase western, high-energy food ('nutrition transition') and avoid physically demanding tasks as symbols of status (30). This results in obesity which in turn is associated with diabetes. Increasing obesity in Bangladesh (31) may also be due to reduced physical activity associated with changing traditional agricultural/domestic works replaced by technology, watching television, and using the internet. Irrespective of the explanation, increasing obesity in Bangladesh suggests that diabetes will increase further with its strengthening economy. A further policy implication is that interventions for diabetes prevention in Bangladesh need to focus on obesity, particularly by reducing the consumption of unhealthy diets and increasing physical activity. This should be given priority as there is evidence that even a modest weight reduction of $5-7 \%$ in high-risk individuals result in a decline in the incidence of diabetes (32) as recent studies demonstrated that the incidence of diagnosed diabetes is stabilizing or declining in many high-income countries since $2010(33,34)$.

Our data also show that diabetes is higher in people with hypertension, which agrees with other studies $(35,36)$. In addition, hypertension is exacerbated by other risk factors such as obesity, advanced age and significantly contributes to micro and macrovascular complications resulting in renal failure and cardiovascular disease $(35,37)$. Pathways through which these complications may occur include insulin resistance, inflammation, and obesity (38). The implication of the strong association of hypertension and diabetes is that efforts are needed in Bangladesh to delay or prevent comorbid hypertension in diabetes through frequent follow-up and aggressive management.

Our study found that awareness, treatment, and control of diabetes are low in Bangladesh. Renewed efforts are needed to increase awareness, treatment, and control to improve diabetes outcomes and reduce/delay complications. Receiving appropriate treatment may be partly influenced by out-of-pocket health costs, large in Bangladesh (39). This places a financial burden on households and has the effect of preventing people from accessing care (health care is viewed as a 'luxury' not a 'necessity') or seeking alternative providers who are cheaper but untrained and cause adverse effects (40). Such barriers need to be addressed as they may undermine diabetes prevention efforts. 
The strengths of the study are that it used a large, nationally representative dataset suggesting the findings have external validity. A further strength is that clinical variables, including FBG, blood pressure, body weight, and height, were measured using high-quality techniques. The WHO criteria for the classification of diabetes and prediabetes were used, while hypertension was defined using the seventh report of the Joint National Committee on Prevention, Detection, Evaluation, and Treatment of high blood pressure criteria. Our multilevel mixed-effects Poisson regression corrects the overestimation of the effects size produced by conventional logistic regression employed in cross-sectional studies and increased the precision of the findings. However, this was a cross-sectional study, which limits our ability to infer causal relationships. The oral glucose tolerance test (OGTT) or $\mathrm{HbA1C}$ tests are the gold standards for diagnosing type 2 diabetes. However, using these gold standards to diagnose diabetes was not possible for population-level data in the context of a resource-poor country. A further limitation was that diabetes medication use was self-reported. Dietary and physical activity data were not collected and so could not be controlled for in the analysis.

\section{Conclusions}

This study implies that efforts to control diabetes and prediabetes in Bangladesh need to be strengthened and optimized, investing further resources. Given that diabetes and prediabetes are preventable diseases by modifying diet and physical activities, Bangladesh needs to intensify its efforts to implement diabetes prevention. This may require the health care system changes in which non-communicable disease prevention is prioritized and household medical care payments reviewed to reduce out-of-pocket expenses. These measures will be worth the investment as they will maximize access to high-quality public health programs. Our analysis further implies that diabetes prevention should focus on reducing obesity and managing hypertension, suggesting that their management will bring the greatest benefits. Without effective preventive measures, diabetes will continue to increase in Bangladesh.

Diabetes and prediabetes affect a substantial proportion (over one-quarter) of the Bangladeshi adult population. Despite worldwide recognition of the increasing burden of diabetes in LMICs and widespread awareness of the need for prevention through lifestyle interventions, these conditions remain a significant public health burden in Bangladesh. Along with obesity and hypertension management, newer approaches to prevention are needed, which address obesogenic environments. These will include creating walkable neighborhoods, encouraging healthy food choices in schools, and workplaces, motivating physical activity and supporting active transport. These should be part of policies to address non-communicable diseases, including diabetes, in Bangladesh.

\section{Acknowledgments}

The authors thank MEASURE DHS for granting access to the BDHS 2017-18 data.

\section{Authors' contributions}


medRxiv preprint doi: https://doi.org/10.1101/2021.01.26.21250519; this version posted August 4, 2021. The copyright holder for this preprint

(which was not certified by peer review) is the author/funder, who has granted medRxiv a license to display the preprint in perpetuity.

All rights reserved. No reuse allowed without permission.

Conception and design: RMI, MBH; Data curation and formal analysis: $M N K, R M I, M B H, J R$; Data interpretation: RMI, MNK, JO, JR, DJM, EC, NK, MBH; Manuscript draft: RMI, JO, MNK, $\mathrm{MBH}$; Reviewed and edited the final manuscript: RMI, MNK, JO, JR, DJM, EC, NK, MBH.

\section{Competing interests}

The authors declare that they have no competing interests.

\section{References}

1. Lin $X, X u Y, X P$, Jingya $X u J$, Ding $Y$, Sun $X$, et al. Global, regional, and national burden and trend of diabetes in 195 countries and territories: an analysis from 1990 to 2025. Nature. 2020;10:14790.

2. Baena-Díez JM, Peñafiel J, Subirana I, Ramos R, Elosua R, Marín-Ibañez A, et al. Risk of cause-specific death in individuals with diabetes: a competing risks analysis. Diabetes Care. 2016;39(11):1987-95.

3. Saeedi P, Petersohn I, Salpea P, Malanda B, Karuranga S, Unwin N, et al. Global and regional diabetes prevalence estimates for 2019 and projections for 2030 and 2045: Results from the International Diabetes Federation Diabetes Atlas. Diabetes research and clinical practice. 2019;157:107843.

4. International Diabetes Federation. IDF Diabetes Atlas, 9th edn. Brussels. Belgium: 2019. Available at: https://www.diabetesatlas.org, accessed 18 November 2020; 2019.

5. Akter S, Rahman MM, Abeb SK, Sultanac P. Prevalence of diabetes and prediabetes and their risk factors among Bangladeshi adults: a nationwide survey. Bull World Health Organ. 2014;92:204-13A.

6. National Institute of Population Research and Training (NIPORT), and ICF. 2020. Bangladesh Demographic and Health Survey 2011. Dhaka, Bangladesh, and Rockville, Maryland, USA: NIPORT and ICF.

7. National Institute of Population Research and Training (NIPORT), and ICF. 2020. Bangladesh Demographic and Health Survey 2017-18. Dhaka, Bangladesh, and Rockville, Maryland, USA: NIPORT and ICF.

8. Saquib N, Saquib J, Ahmed T, Khanam MA, Cullen MR. Cardiovascular diseases and type 2 diabetes in Bangladesh: a systematic review and meta-analysis of studies between 1995 and 2010. BMC public health. 2012;12(1):434.

9. Al Kibria GM, Hashan MR, Hossain MM, Zaman SB, Stennett CA. Clustering of hypertension, diabetes and overweight/obesity according to socioeconomic status among Bangladeshi adults. Journal of biosocial science. 2021;53(2):157-66.

10. Chowdhury MAB, Uddin MJ, Khan HM, Haque MR. Type 2 diabetes and its correlates among adults in Bangladesh: a population based study. BMC Public Health. 2015;15(1):1-11.

11. Harshfield E, Chowdhury R, Harhay MN, Bergquist $H$, Harhay MO. Association of hypertension and hyperglycaemia with socioeconomic contexts in resource-poor settings: the Bangladesh Demographic and Health Survey. International journal of epidemiology. 2015;44(5):1625-36.

12. Swasey K, Al Kibria GM, Stafford K, editors. A conservative approach to estimating the socioeconomic factors associated with diabetes in Bangladesh. APHA's 2018 Annual Meeting \& Expo (Nov 10-Nov 14); 2018: American Public Health Association.

13. Al Kibria GM. Prevalence and Factors Associated with Diabetes among Bangladeshi Adults: An Analysis of Demographic and Health Survey 2017-18. Diabetes Epidemiology and 
Management. 2021;2:100012.

14. Barros AJ, Hirakata VN. Alternatives for logistic regression in cross-sectional studies: an empirical comparison of models that directly estimate the prevalence ratio. BMC medical research methodology. 2003;3(1):21.

15. Tamhane AR, Westfall AO, Burkholder GA, Cutter GR. Prevalence odds ratio versus prevalence ratio: choice comes with consequences. Statistics in medicine. 2016;35(30):5730-5.

16. United Nations, Department of Economic and Social Affairs, Population Division. World Population Prospects 2019: Highlights (ST/ESA/SER.A/423), available at: https://population.un.org/wpp/Publications/Files/WPP2019_Highlights.pdf accessed on 24 Jan 2021. 2019.

17. World Health Organization. Definition and diagnosis of diabetes mellitus and intermediate hyperglycaemia: report of a WHO/IDF consultation. 2006.

18. World Health Organization Expert Consultation. Appropriate body-mass index for Asian populations and its implications for policy and intervention strategies. Lancet (London, England). 2004;363(9403):157-63.

19. Chobanian AV, Bakris GL, Black HR, Cushman WC, Green LA, Izzo Jr JL, et al. The seventh report of the joint national committee on prevention, detection, evaluation, and treatment of high blood pressure: the JNC 7 report. Jama. 2003;289(19):2560-71.

20. Rutsein SO, Johnson K. The DHS Wealth Index. DHS Comparative Reports No. 6. Calverton, Maryland, USA: ORC Macro. Available at http://dhsprogram.com/publications/publication-cr6-comparative-reports.cfm. Accessed November 07, 2015.; 2004.

21. Yelland LN, Salter AB, Ryan P. Performance of the modified Poisson regression approach for estimating relative risks from clustered prospective data. American journal of epidemiology. 2011;174(8):984-92.

22. O'Connell AA, McCoach DB. Multilevel modeling of educational data: IAP; 2008.

23. Von Elm E, Altman DG, Egger M, Pocock SJ, Gøtzsche PC, Vandenbroucke JP. The Strengthening the Reporting of Observational Studies in Epidemiology (STROBE) statement: guidelines for reporting observational studies. Annals of internal medicine. 2007;147(8):5737.

24. NCD Risk Factor Collaboration. Worldwide trends in diabetes since 1980: a pooled analysis of 751 population-based studies with 4.4 million participants. . Lancet. 2016;387(10027).

25. Zaman $M$, Ullah $A$, Bhuiyan $M$, Karim $M$. Moniruzzaman and Rahman SMA. Noncommunicable Disease Prevention and Control Situation in a Primary Health Care Setting of Bangladesh: Design and Baseline Findings of an Intervention Chronic Dis Int. 2016;3(1):1021.

26. Mohan V, Deepa M, Anjana RM, Lanthorn H, Deepa R. Incidence of diabetes and prediabetes in a selected urban south Indian population (CUPS-19). . J Assoc Physicians India. 2008;56:152-7.

27. Chen Y, Zhang X-P, Yuan J, Cai B, Wang X-L, Wu X-L, et al. Association of body mass index and age with incident diabetes in Chinese adults: a population-based cohort study. BMJ open. 2018;8(9):e021768.

28. World Bank. https://data.worldbank.org/indicator/SP.POP.TOTL?locations=BD. 2021.

29. Barnes AS, Coulter SA. The Epidemic of Obesity and Diabetes Trends and Treatments Tex Heart Inst J. 2011;38(2):142-4. 
medRxiv preprint doi: https://doi.org/10.1101/2021.01.26.21250519; this version posted August 4, 2021. The copyright holder for this preprint (which was not certified by peer review) is the author/funder, who has granted medRxiv a license to display the preprint in perpetuity.

All rights reserved. No reuse allowed without permission.

30. Pampel FC, Denney JT, Krueger PM. Obesity, SES, and Economic Development: A Test of the Reversal Hypothesis. Social Science and Medicine. 2012;74(7):1073-81.

31. Banik S, Rahman M. Prevalence of overweight and obesity in Bangladesh: a systematic review of the literature. Current obesity reports. 2018;7(4):247-53.

32. Knowler WC, Barrett-Connor E, Fowler SE, Hamman RF, Lachin JM, Walker EA, et al. Reduction in the incidence of type 2 diabetes with lifestyle intervention or metformin. The New England journal of medicine. 2002;346(6):393-403.

33. Magliano DJ, Chen L, Islam RM. Multi-country analysis of trends in the incidence of diagnosed diabetes from 22 million diagnoses in higher- income settings. The Lancet Diabetes \& Endocrinology. 2021.

34. Magliano DJ, Islam RM, Barr EL, Gregg EW, Pavkov ME, Harding JL, et al. Trends in incidence of total or type 2 diabetes: systematic review. bmj. 2019;366:15003.

35. Lastra G, Syed S, Kurukulasuriya LR, Manrique C, Sowers JR. Type 2 diabetes mellitus and hypertension: an update. Endocrinology and Metabolism Clinics. 2014;43(1):103-22.

36. Suh D-C, Kim C-M, Choi I-S, Plauschinat CA, Barone JA. Trends in blood pressure control and treatment among type 2 diabetes with comorbid hypertension in the United States: 1988-2004. Journal of hypertension. 2009;27(9):1908-16.

37. Simonson DC. Etiology and prevalence of hypertension in diabetic patients. Diabetes care. 1988;11(10):821-7.

38. Cheung BM, Li C. Diabetes and hypertension: is there a common metabolic pathway? Current atherosclerosis reports. 2012;14(2):160-6.

39. Afroz A, Habib SH, Chowdhury HA, Paul D, Shahjahan Md, Hafez A, et al. Healthcare cost of type 2 diabetes mellitus in Bangladesh: a hospital-based study. Int J Diabetes Dev Ctries. 2016;36(2):235-41.

40. Khan JA, Ahmed S, Sultana M, Sarker AR, Chakrovorty S, Rahman MH, et al. The effect of a community-based health insurance on the out-of-pocket payments for utilizing medically trained providers in Bangladesh. International health. 2020;12(4):287-98. 
medRxiv preprint doi: https://doi.org/10.1101/2021.01.26.21250519; this version posted August 4, 2021. The copyright holder for this preprint (which was not certified by peer review) is the author/funder, who has granted medRxiv a license to display the preprint in perpetuity.

All rights reserved. No reuse allowed without permission.

Figure 1: Schematic representation of the sampling procedure of the Bangladesh Demographic and Health Survey, 2017-18

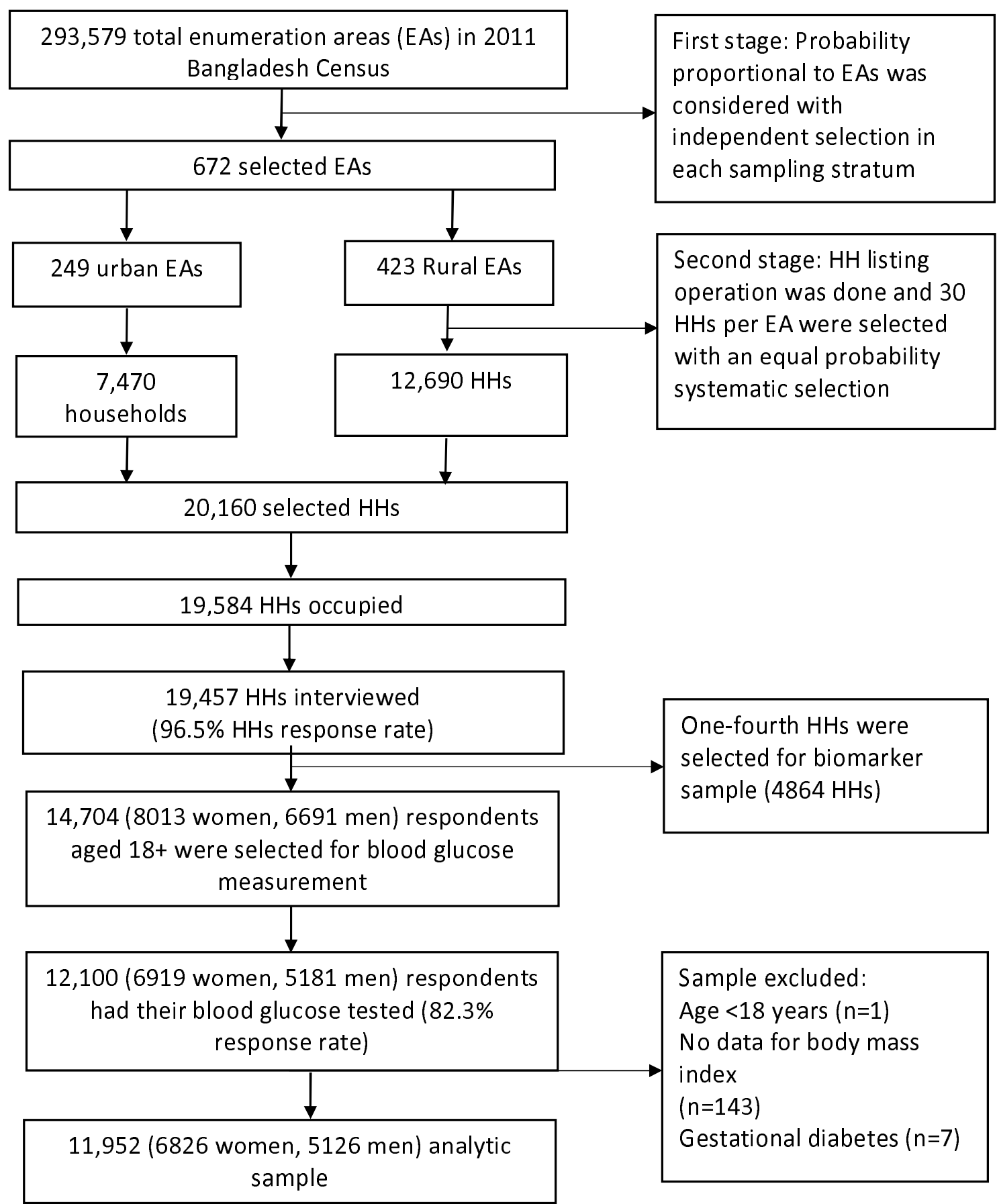

